\title{
Improving the selectivity to polar vapors of OFET-based sensors by using the transfer characteristics hysteresis response
}

\author{
Peter Lienerth, Sadiara Fall, Patrick Lévêque, Ugur Soysal, Thomas Heiser* \\ Laboratoire ICube, Département ESSP, Université de Strasbourg, CNRS, 23 rue du Loess, Strasbourg Cedex 2, France
}

\section{A R T I C L E I N F O}

\section{Article history:}

Received 29 June 2015

Received in revised form 2 November 2015

Accepted 3 November 2015

Available online 10 November 2015

\section{Keywords:}

OFET

Hysteresis

Poly-3-hexylthiophene

Gas sensors

Ethanol

Acetone

\begin{abstract}
A B S T R A C T
Sensing of polar volatile organic compounds by generic organic-field-effect transistors has been achieved by monitoring both, the drain current at a fixed gate voltage and the charge carrier mobility. In this paper we show that the hysteresis of the transfer-characteristics of P3HT-based OFETs can be used as an additional sensing parameter for the detection of polar vapors. The response of the hysteresis to polar vapors is marked by a reversible increase at a time-scale similar to the previously reported parameters. By supplementing the responses of on-current and mobility with the hysteresis-response, an improvement of the device selectivity to ethanol and acetone vapor is achieved.
\end{abstract}

(C) 2015 Elsevier B.V. All rights reserved.

\section{Introduction}

The increase of conductivity in polymer films during exposure to gaseous analytes, first reported in the groundbreaking work of Shirakawa, Louis, Mac Diarmid and Heeger, initiated an intense research activity in organic conductors and semiconductors (OS) $[1,2]$. Today, the performance of OS and the inexpensive production procedures have led to commercially available OLED displays and photovoltaic cells for demonstration purposes. The sensitivity of OS to gases, however, has been less investigated, though inexpensive large-scale production of gas sensors is expected to enable new applications in environmental monitoring, food spoilage detection and health industry. Solution processable organic semiconductors are promising candidates as they have been shown to exhibit fast and reproducible responses at room temperature [3] and respond to various analyte gases, ranging from volatile organic compounds $[4,5]$ over airborne pollutants as $\mathrm{NO}_{2}$ [6] and hazardous gases like ammonia [7-9] to explosives, which require an utterly weak detection limit [10-13]. This large number of detectable gases enables various applications for OS in gas sensors on one hand, but challenges the selectivity of the devices, on the other hand.

\footnotetext{
* Corresponding author.

E-mail addresses: Peter.lienerth@horiba.com (P. Lienerth), sadiara.fall@unistra.fr (S. Fall), patrick.leveque@unistra.fr (P. Lévêque), ugursoysal@yahoo.com (U. Soysal), thomas.heiser@unistra.fr (T. Heiser).
}

To improve the selectivity of organic semiconductor-based gas sensors, several methodologies have been reported. Specific functional groups can be covalently grafted to the conjugated backbone and have been shown to increase the selectivity and even to enable chiral recognition in the tens of ppm range [14,15]. However, most organic semiconductors do not show such a discriminative power and demand different approaches. Sensor arrays combine multiple sensors composed of different generic organic semiconductors in a single platform. As each material responds differently to a given analyte, the entire array generates unique signal patterns that allow the identification of the impinging gaseous species without particular receptors for the analytes $[16,17]$. Most sensor arrays employ chemresistor structures, which are facile to produce but only respond to analytes by resistance changes. While this oneparameter response of a chemiresistor impedes the identification of the analyte by the response of a single device, organic field-effect transistors (OFETs) deliver more complex responses that render selectivity also to single devices. Torsi et al. have shown that the combined responses of field effect mobility $\mu$, threshold voltage $V_{\text {th }}$, on-current $I_{\text {on }}$ and off-current $I_{\text {off }}$ allow to distinguish analyte vapors [18]. As these OFET parameters are differently affected by the OS-analyte interactions, the multiparameter data set of an OFET device enables fingerprint-like response patterns which allow the identification of various analytes.

Another parameter of the OFET characteristics that has been reported to show changes during exposure to gas is the hysteresis of the transfer-characteristics which describes the current 
difference measured between the forward and the reverse sweeps of the gate voltage. Gu and Kim studied the hysteresis in pentacenebased OFETs during exposure to moisture and reported an increase due to moisture-induced trapping [19,20]. Tang reported a not completely reversible increase of the hysteresis in dendrimer-based OFETs [12]. However, to the best of our knowledge, a systematic investigation about the application of the hysteresis as a parameter for the detection of vapors with OFET-based sensors has not yet been reported.

In this paper we use the well-studied non-functionalized os poly(3-hexylthiophene) (P3HT) for the detection of ethanol and acetone vapors to investigate the performance of the hysteresis as a sensing parameter. Using OS-based sensors for the detection of alcohol is of particular interest, as many inorganic sensors are either poisoned by alcohols or require high temperatures of a few $100^{\circ} \mathrm{C}$ for the detection. Acetone $\left(\mathrm{C}_{3} \mathrm{H}_{6} \mathrm{O}\right)$ was chosen for comparison, as it shows a comparable size and a $\mathrm{C}-\mathrm{O}$-based backbone as ethanol $\left(\mathrm{C}_{2} \mathrm{H}_{6} \mathrm{O}\right)$, while its polar moment is almost twice as high (ethanol: $1.69 \mathrm{D}$, acetone: $2.91 \mathrm{D}$ ). At ambient temperatures the response of OFETs to ethanol has already been reported by several groups, as a decrease in current and mobility upon exposure $[16,21]$. Temperature dependent measurements with pentacene OFETs revealed an increase of the activation energy for hopping during exposure to ethanol vapor, as origin of the mobility decrease. It has been suggested that the carrier trap depths are altered in presence of polar molecules due to electrostatic disorder induced by the analyte dipoles [22]. The interaction mechanisms of acetone have not yet been reported but the high polar moment can be expected to similarly contribute to charge carrier trapping. As acetone is also a weak Lewis base, due to the carbonyl-group, the formation of hydrogen bonds is also a possible interaction mechanism.

By simultaneously tracking on-current, mobility and hysteresis in a single transfer-characteristics measurement, we show that the performance of the hysteresis is comparable to the behavior of current and mobility in terms of response/recovery time, sensitivity and stability while the additional information, gained by the utilization of the hysteresis, will be shown to increase the selectivity of the OFET device. Finally, the device multi-parametric response to water vapor is briefly described in order to assess the sensor utilization in ambient conditions.

\section{Materials and methods}

The gas sensors were fabricated on bottom gate bottom contact field-effect transistor substrates, purchased from the Fraunhofer-Institute for Photonic Microsystems [23]. N-doped silicon substrates serve as gate electrode and are covered by a $230 \mathrm{~nm}$ thick thermal oxide layer as dielectric. The interdigitated source and drain electrodes consist of a $10 \mathrm{~nm}$ thick ITO adhesion layer, topped with $30 \mathrm{~nm}$ gold. The transistor substrates were provided with several channel lengths out of which 5 and $10 \mu \mathrm{m}$ devices with a $1 \mathrm{~cm}$ channel width are shown in this article. The substrates were shipped with a protective layer which was removed prior to the deposition of the OS by ultrasonic baths performed in the following order: soap and distilled water, acetone and finally isopropanol. To remove any residual contaminants the substrates underwent an UV-ozone treatment before being transferred into a nitrogen-filled glove box system.

Poly(3-hexylthiophene) (P3HT) was ordered from Riecke Metals, Inc. and has an averaged molecular weight $M_{\mathrm{w}}$ of $60-75 \mathrm{~kg} / \mathrm{mol}$, and a regioregularity above $96 \%$. Thin films were deposited by spin-coating from 1,2-dichlorobenzene solutions with concentrations of $1 \mathrm{mg} / \mathrm{ml}$ at $2000 \mathrm{rpm}$ for $180 \mathrm{~s}$. After deposition, devices were dried in $<10^{-5}$ mbar vacuum for $2 \mathrm{~h}$ at least and permanently kept in a nitrogen atmosphere. This resulted in film thicknesses of approximately $10 \mathrm{~nm}$, measured by a DEKTAK profilometer in vicinity to the outermost source and drain electrodes.

To perform gas sensing experiments the OFETs were contacted through feedthroughs in a hermetically sealed stainless steel chamber (detector chamber) with PTFE lining on the inside. The detector chamber is connected to two tanks, each containing a different analyte source. Two Brooks mass flow controllers (MFC) are used, out of which one delivers pure nitrogen directly to the OFET chamber for purging while the other is used to control the nitrogen flux through the analyte tanks where it serves as a carrier gas for the analyte vapor.

To create analyte vapor, the liquid phase of the volatile analyte evaporates from a vial in a chamber where nitrogen carrier gas is introduced. The outlet of this analyte chamber can either be directed to the detector chamber or to the exhaust. Before exposure the analyte chamber is purged for $20 \mathrm{~min}$ at least, to establish equilibrium between incoming nitrogen and evaporating analyte. The concentration of the analyte vapors in the carrier gas was approximated by measuring the mass loss of the liquid phase after purging the chamber at a constant flow rate for more than $12 \mathrm{~h}$. The used flow rate was $3 \mathrm{l} / \mathrm{h}$, as it was also used during the experiment. The mass loss was averaged out over time and converted to molecular concentration by dividing by the corresponding molecular weight.

For exposure, the flow rate of the MFC used to purge the detector chamber was reduced by $3 \mathrm{l} / \mathrm{h}$ and the flow from the stabilized analyte-source was added. After exposure the flow from the analyte source was directed to the exhaust and the flow rate of the first MFC, which directly goes to the detector, was changed back to the initial value. To achieve different concentrations the flow rate of the first MFC was changed once the device stabilized.

As experiments were performed at room temperature the actual concentrations may differ from the values derived by the calibration procedure. Fluctuations of $1{ }^{\circ} \mathrm{C}$, typically occurring during a day, change the saturated vapor pressure, and therefore also the generated concentrations by up to 7\% [24]. However, as this communication focuses on the comparison of different OFET parameters which were extracted from a single measurement of the transfer characteristics of the investigated OFETs, the accuracy of the concentrations is not a limitation to the derived results.

Electrical characterization during sensing was conducted in the dark at room temperature under a constant flow of nitrogen or the nitrogen/analyte mixture.

The transfer-characteristics measurements were performed with a 4156 C Agilent semiconductor parameter analyzer. Devices were measured with a staircase gate voltage sweep from 20 to $-60 \mathrm{~V}$ and reverse. In the examples shown, the drain voltage was set to $-15 \mathrm{~V}$. Between adjacent measurements of the transfercharacteristics all terminals were grounded for $10 \mathrm{~s}$ to reduce bias-stress effects and enable the data transfer to the piloting LabVIEW program. This waiting time between two measurements was used to change the flow rates and directions of the MFC, i.e. start and stop exposures. The area of the hysteresis was calculated by summing up the current differences between the forward and the reverse sweeps for every gate voltage step and multiplying the result with the gate voltage step size. Mobility $\mu$ was derived from the linear regime, fitted in the -55 to $-60 V_{\mathrm{g}}$ range according to $\mu=\left(L / W C V_{\mathrm{d}}\right)\left(\partial I_{\mathrm{d}} / \partial V_{\mathrm{g}}\right)$, where $L$ and $W$ are channel length and width of the device, respectively. $C$ is the capacitance per unit area of the $\mathrm{SiO}_{2}$ dielectric, $V_{\mathrm{d}}$ is the applied drain voltage. $I_{\mathrm{d}}$ denotes the drain current and $V_{\mathrm{g}}$ the applied gate voltage.

To reduce interferences from bias stress effects, the devices were continuously measured before the exposures until the drift became negligible in comparison to the response amplitudes of the devices. 


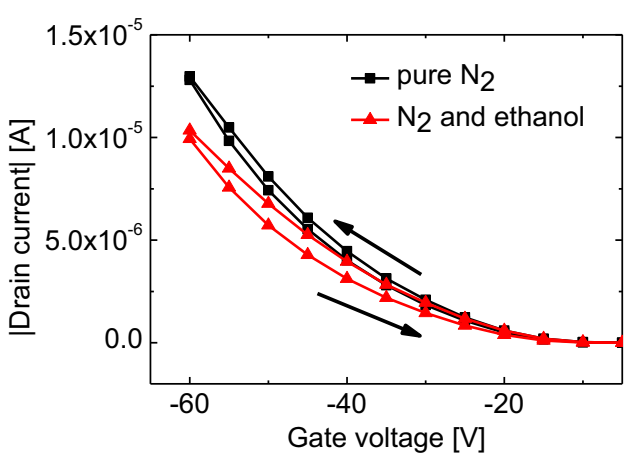

Fig. 1. Transfer-characteristics of a P3HT-OFET with $10 \mu \mathrm{m}$ channel length in nitrogen and during exposure to ethanol vapor. For a better visibility the concentration of ethanol is close to the saturated vapor pressure. Devices were measured with a staircase gate voltage sweep from 20 to $-60 \mathrm{~V}$ and reverse. The drain voltage was set to $-15 \mathrm{~V}$.

\section{Results and discussion}

The transfer characteristics of transistors are measured by keeping source and drain at fixed potentials, while the gate voltage sweeps from the "off" to the "on" regime of the transistor and back to the off regime. The off-to-on sweep is referred to as forward sweep and the on-to-off sweep is called reverse sweep. In an ideal transistor the source-drain current is not affected by the direction of the gate voltage sweep and the currents measured in the forward and the reverse sweeps are similar. In OFET devices, deviations of the current measured in the reverse sweep from the current measured in the forward sweep were frequently reported and are generally referred to as hysteresis effects [25]. A notation for the direction of the deviation was given by Egginger et al., by classifying as lower or higher back sweep current (lower/higher BSC).

The effect of polar vapors on the transfer characteristics of generic OFETs has been described as a decrease of on-current and mobility. Both of these parameters are extracted from the forward sweep of the transfer-characteristics only. Fig. 1 shows the forward and the reverse sweeps of the transfer-characteristics in the onregime of a P3HT OFET, measured in a pure nitrogen atmosphere. As the arrows indicate, the current measured in the reverse sweep is slightly lower compared to the current measured in the forward sweep, leading to a lower BSC hysteresis. The same graph shows also the behavior of the same device during exposure to highly concentrated ethanol vapor. In accordance with previous reports, a decrease of the on-current and slope, which is proportional to the mobility, can be observed. Additionally, the difference between the currents measured in the forward and the reverse sweeps for a given gate voltage, i.e. the hysteresis, has increased during exposure to ethanol. Though only shown for the linear regime in Fig. 1, a similar behavior was also found in the saturation regime. For this work it was decided to focus on the linear regime of the transfer characteristics, as the conductive channel is more homogeneous in this regime. Additionally it should be mentioned that the described behavior was observable for all four channel lengths provided by the purchased substrates.

To check the feasibility of using the hysteresis as additional parameter for ethanol detection, we compared the hysteresis response (estimated as described in the experimental section) to mobility and on-current changes as a function of time and ethanol concentration (Fig. 2).

The device was exposed to high concentrations of 2000 and $2800 \mathrm{ppm}$ for $15 \mathrm{~min}$, while the lower concentrations of 1000 and $500 \mathrm{ppm}$ lasted only $8 \mathrm{~min}$. In accordance with previously reported results, mobility and drain-current show a decrease upon exposure to ethanol which recovers almost completely after withdrawal of the analyte. For the hysteresis response, a similar behavior on the same time-scale can be observed, indicating a comparable performance in terms of response speed and recovery time. To evaluate the reproducibility of the hysteresis response, the exposures from Fig. 2a were performed twice and the relative responses $\left(P_{\text {gas-on }}-P_{\text {gas-off }}\right) / P_{\text {gas-off }} \times 100$ of all three parameters $(P)$ are shown in Fig. 2b. The drain current response, which is the drain current measured at a single gate voltage $\left(V_{g}=-60 \mathrm{~V}\right)$, shows the most stable response, while mobility and hysteresis, both based on the measurements of several points of the transfer-characteristics, are more subjected to noise. As a consequence, though the hysteresis shows an almost 10 times higher percentual response, the sensitivity is intermediate to on-current and mobility. Fig. 2b also shows that all three parameters respond almost linearly with the ethanol concentration. A linear relation between the on-current response and the ethanol concentration was already reported for pentacene and methoxysubstituted poly(triaryl amine) and is favorable as a sensor response, as it facilitates calibration and quantification of the responses [21,26].

One of the most important properties of a sensor is the ability to selectively detect an analyte gas without interference from other components. As it has already been shown by Torsi, utilization of multiple transistor parameters enables the recognition of a)

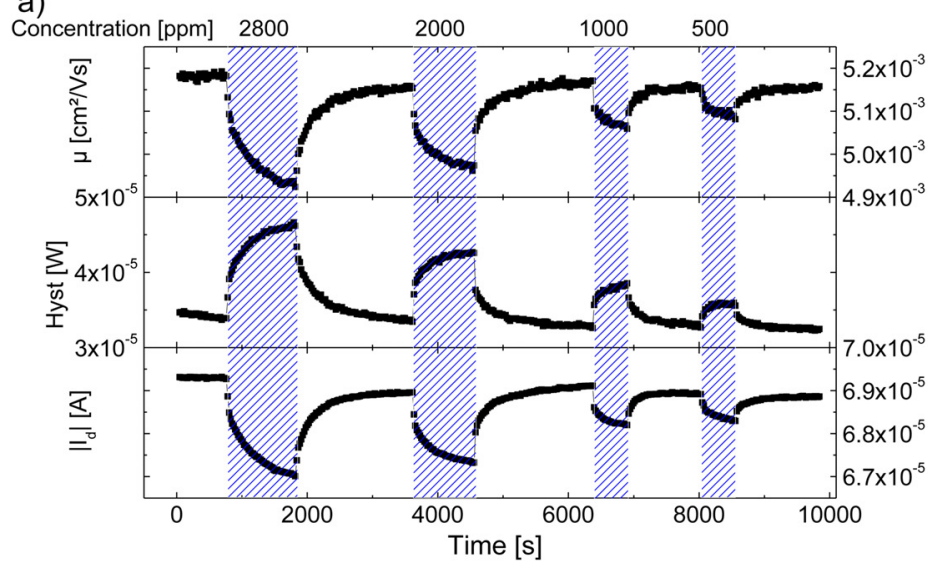

b)

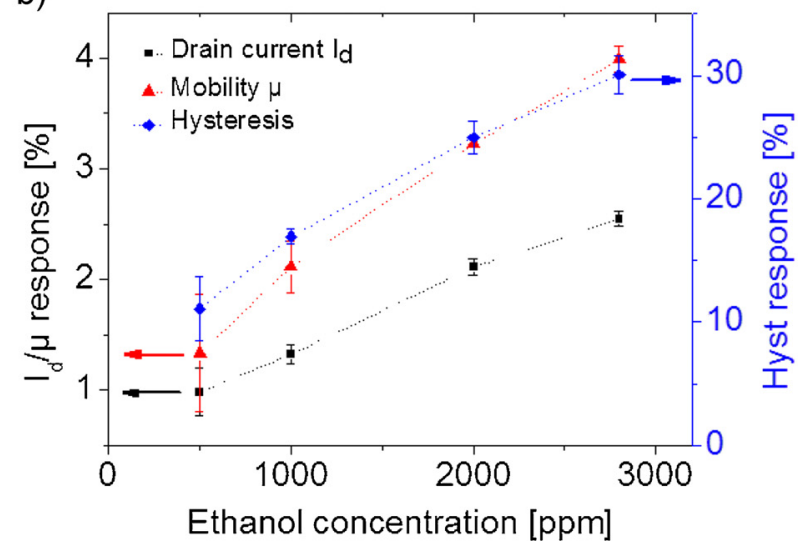

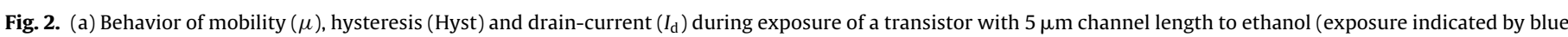

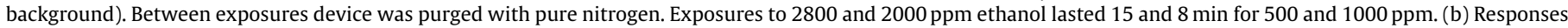

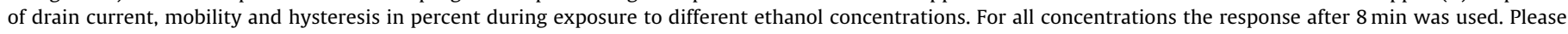
note, only the hysteresis response is referring to the ordinate on the right side. 

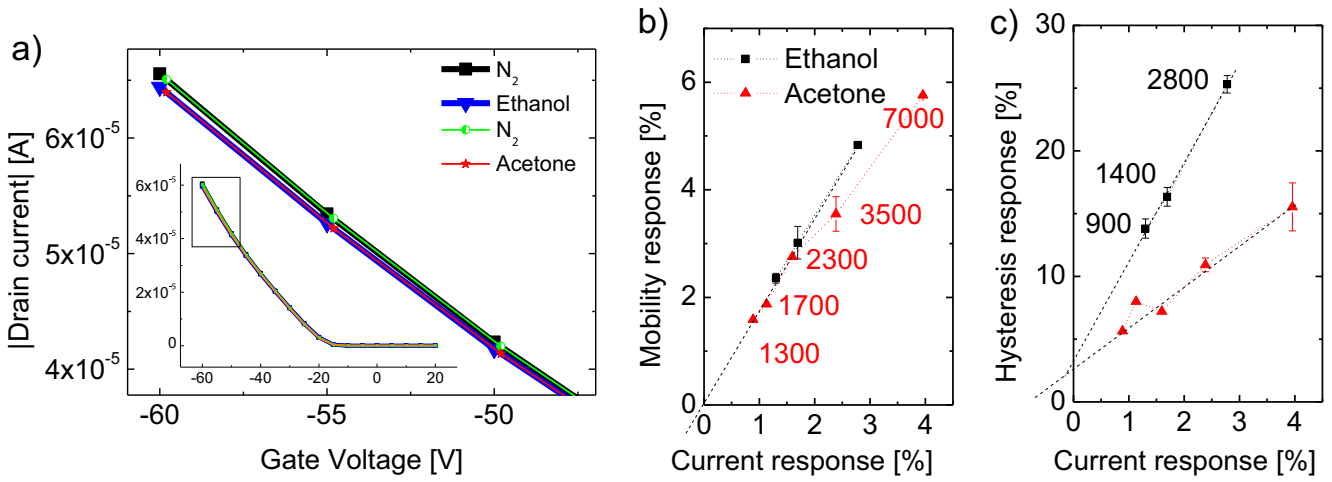

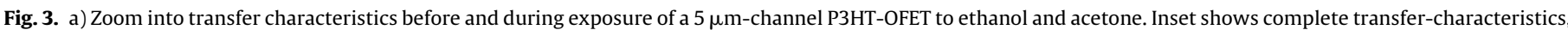

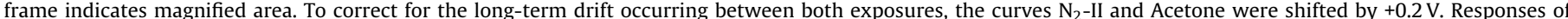

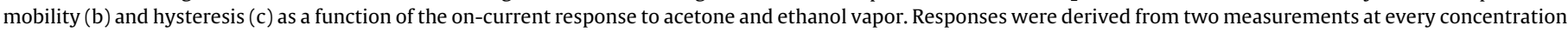

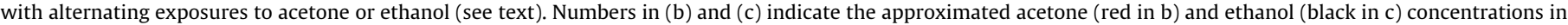
ppm, respectively.

molecular species by a single OFET device [18]. To demonstrate the gain in selectivity provided by the additional utilization of the hysteresis P3HT-based OFETs were exposed to two vapors which give rise to responses indistinguishable by conventional parameters as drain current, mobility and threshold voltage. Fig. 3a depicts the forward sweep of the transfer characteristics during exposure to $1400 \mathrm{ppm}$ ethanol and $2300 \mathrm{ppm}$ acetone and the corresponding curves under $\mathrm{N}_{2}$ measured immediately before the exposure. To correct for the long-term drift of the threshold voltage the second measurement in $\mathrm{N}_{2}$ (N2-II) and the subsequent exposure to acetone were shifted by $+0.2 \mathrm{~V}$ to match both curves under $\mathrm{N}_{2}$. The curves recorded $8 \mathrm{~min}$ after onset of the exposures to ethanol and acetone were intentionally chosen so as to fit to each other. This allows to demonstrate that changes in drain current and mobility are identical for both gases, making a selective detection impossible with the conventional parameters.

Fig. 3b compares the percentual response of mobility versus drain current for different concentrations of ethanol and acetone. To ensure the responses are not affected by drift-induced changes the devices were first exposed to the set of concentrations shown for ethanol, followed by the concentrations indicated for acetone and then again exposed to the concentrations for ethanol and acetone. Variations of the responses are shown by error bars in the graphs.

As it can be seen, both gases lead to similar response-patterns for the parameters mobility and drain-current, making their usage unsuitable for the discrimination of acetone and ethanol in the concentration ranges investigated. The hysteresis response is shown as a function of the drain current in Fig. 3c. Though all parameters in Fig. 3b and c were extracted from the same measurements, Fig. 3c shows two distinctive curves for the analytes. For comparable current responses ethanol causes an approximately two times higher hysteresis response than acetone, allowing the identification of the analytes over the whole concentration range measured. Similar results obtained on OFETs with different channel lengths are given in the Supporting Information.

Though the higher polar moment of acetone could be expected to imply stronger responses, these results point out a lower sensitivity of the device toward acetone, which is in agreement with the results derived from chemiresistor devices described by $\mathrm{Li}$ et al. [27]. A reduced adsorption and/or absorption of the highly polar acetone by the non-polar P3HT, are likely to give rise to the observed behavior.

The comprehensive overview of hysteresis-effects in OFETs given by Egginger [25] suggested three phenomena to be at the origin of a lower Back-Sweep Current (BSC). Mobile ions in the semiconductor would be affected by an applied gate voltage and migrate toward or away from the conductive channel, depending on the type of charge carriers and applied gate bias. Positive ions in P3HT could be attracted toward the dielectric by the negative gate voltage in the on-regime of the transistor. In vicinity to the conductive channel the positive ions screen the electric field from the gate and in consequence, reduce the number of free charge carriers during the back-sweep of the gate voltage. However, the dissociation of ethanol or acetone molecules has not been reported in transistor-devices, yet, and the applied gate-voltages of $60 \mathrm{~V}$ maximum, are not expected to give rise to the observed phenomenon.

A second explanation given by Egginger is trapping of minority charge carriers, which appears while the device is biased in the off-regime. In the case of P3HT, this would mean electron traps can be filled while the device is biased positively, at the onset of the measurement. When sweeping to the on-regime of the device the negatively charged traps induce additional holes into the conductive channel which leads to excess holes in the device. As trapped electrons are released over time, fewer electrons are trapped during the reverse sweep of the gate voltage leading to a lower total drain current. In order to understand if electron traps contribute to the hysteresis increase observed in polar vapors the gate sweep range was limited to negative biases and compared to responses observed for a sweep range from 20 to $-40 \mathrm{~V}$. The relative current and hysteresis responses shown in Table 1 are almost identical for both parameters and show rather a decrease of the hysteresis response when the device is biased positively, indicating that electron traps do not significantly contribute to the response of the hysteresis to polar vapors.

A third mechanism suggested by Egginger is trapping of majority charge carriers. Holes, trapped during the forward sweep of the transfer characteristics, remain trapped during the reverse sweep. This effect decreases the number of free charge carriers in the reverse sweep and reduces the drain current. Temperature-dependent measurements indicated majority charge-carrier trapping as origin of the response of pentacene

Table 1

Responses of $10 \mu \mathrm{m}$ channel P3HT transistor to 800 ppm ethanol:.

\begin{tabular}{lll}
\hline Sweep range $[V]$ & Current response [\%] & Hysteresis response [\%] \\
\hline 0 to -40 & $2.4 \pm 0.2$ & $15,6 \pm 0.9$ \\
+20 to -40 & $2.4 \pm 0.1$ & $14,6 \pm 0.4$ \\
\hline
\end{tabular}


OFETs to ethanol vapor [22]. Based on these conclusions and our observations, we believe that the observed increase of the hysteresis is due to enhanced trapping of majority charge carriers. Furthermore, preliminary measurements of the transient current indicate that the differences in the hysteresis response, observed during exposure to ethanol and acetone, may be caused by alterations of the trapping kinetics by the respective analyte vapor. More detailed investigations of the trapping kinetics will be discussed elsewhere.

Finally, to assess the sensor utilization in ambient conditions, the device response to humidity was briefly investigated. The multi-parameter response before, during and after exposure to $11000 \mathrm{ppm}$ of $\mathrm{H}_{2} \mathrm{O}$ in nitrogen flow $(3 \mathrm{l} / \mathrm{h}$ ) are shown in the supporting information. A decrease in drain current and charge carrier mobility as well as an increase in hysteresis are observed. The sensitivity of each parameter to water is close to the response observed for acetone. However, the change in hysteresis is found to be less reversible (on the time scale of the measurements, i.e. tens of seconds) than the corresponding response to acetone. After a prolonged (a few hours) waiting time at zero bias in a nitrogen atmosphere, the sensor displayed a similar response to ethanol exposure than prior to the humidity test. These preliminary results indicate that the exposure to low humidity levels is not detrimental to the sensor. Additionally, they suggest that the hysteresis evolution upon exposure to the analyte could further enhance the selectivity of the sensor. However more experiments are needed to confirm this corollary.

\section{Conclusions}

In this paper we introduced the hysteresis of the transfercharacteristics as a new parameter for the detection of polar vapors. Derived from the same measurement, its behavior as a gas-sensing parameter has shown properties comparable to the previously used parameters on-current and mobility. Additionally an improvement of the selectivity has been evidenced with ethanol and acetone vapors if the hysteresis is used in combination with the on-current or mobility response.

\section{Acknowledgments}

This work enrolled in the project TRANSFILSEN which was funded by the Agence Nationale de la Recherche. The author is very grateful to Stéphane Roques, Nicolas Zimmermann, Pascal Leindecker and Desbordes Thibault at the ICUBE laboratory Strasbourg for technical support and to Jérôme Heitz for discussions and stimulation. Professor Jean-Pierre Lère-Porte at IS2M in Montpellier is being acknowledged for the vital contribution to this work.

\section{Appendix A. Supplementary data}

Supplementary data associated with this article can be found, in the online version, at http://dx.doi.org/10.1016/j.snb.2015.11.012.

\section{References}

[1] H. Shirakawa, E.J. Louis, A.G. MacDiarmid, C.K. Chiang, A.J. Heeger, Synthesis of electrically conducting organic polymers: halogen derivatives of polyacetylene, J. Chem. Soc. Chem. Commun. (16) (1977) 578-580, http://dx. doi.org/10.1039/C39770000578.

[2] G. Meller, T. Grasser, Organic Electronics, Springer, Berlin Heidelberg, 2010.

[3] J.B. Chang, V. Liu, V. Subramanian, K. Sivula, C. Luscombe, A. Murphy, J. Liu, J.M.J. Fréchet, Printable polythiophene gas sensor array for low-cost electronic noses, J. Appl. Phys. 100 (1) (2006), http://dx.doi.org/10.1064/1. 2208743, 014506.

[4] B. Li, G. Sauvé, M.C. Iovu, M. Jeffries-El, R. Zhang, J. Cooper, S. Santhanam, L. Schultz, J.C. Revelli, A.G. Kusne, T. Kowalewski, J.L. Snyder, L.E. Weiss, G.K.
Fedder, R.D. McCullough, D.N. Lambeth, Volatile organic compound detection using nanostructured copolymers, Nano Lett. vol. 6 (8) (2006) 1598-1602, http://dx.doi.org/10.1021/nl060498o.

[5] A. Zanelli, S. Bassini, M. Giorgetti, Y. Li, M.J. Yang, Chemiresistors for ethanol detection in hydrocarbons, Sens. Actuators B Chem. 148 (1) (2010) 147-152, http://dx.doi.org/10.1016/j.snb.2010.04.037.

[6] a. Das, R. Dost, T. Richardson, M. Grell, J.J. Morrison, M.L. Turner, A Nitrogen dioxide sensor based on an organic transistor constructed from amorphous semiconducting polymers, Adv. Mater. vol. 19 (22) (2007) 4018-4023, http:// dx.doi.org/10.1002/adma.200701504.

[7] J. Yu, X. Yu, L. Zhang, H. Zeng, Ammonia gas sensor based on pentacene organic field-effect transistor, Sens. Actuators B Chem. 173 (2012) 133-138, http://dx.doi.org/10.1016/j.snb.2012.06.060.

[8] S. Tiwari, A.K. Singh, L. Joshi, P. Chakrabarti, W. Takashima, K. Kaneto, R. Prakash, Poly-3-hexylthiophene based organic field-effect transistor: Detection of low concentration of ammonia, Sens. Actuators B Chem. vol. 171-172 (2012) 962-968, http://dx.doi.org/10.1016/j.snb.2012.06.010.

[9] L. Li, P. Gao, M. Baumgarten, K. Müllen, N. Lu, H. Fuchs, L. Chi, High performance field-effect ammonia sensors based on a structured ultrathin organic semiconductor film, Adv. Mater. 25 (July (25)) (2013) 3419-3425, http://dx.doi.org/10.1002/adma.201301138.

[10] R.S. Dudhe, J. Sinha, A. Kumar, V.R. Rao, Polymer composite-based OFET sensor with improved sensitivity towards nitro based explosive vapors, Sens. Actuators B Chem. vol. 148 (1) (2010) 158-165, http://dx.doi.org/10.1016/j. snb.2010.04.022.

[11] J. Huang, T.J. Dawidczyk, B.J. Jung, a.J. Sun, F. Mason, H.E. Katz, Response diversity and dual response mechanism of organic field-effect transistors with dinitrotoluene vapor, J. Mater. Chem. 20 (13) (2010) 2644, http://dx.doi. org/10.1039/B916037J.

[12] G. Tang, S.S.Y. Chen, K.H. Lee, A. Pivrikas, M. Aljada, P.L. Burn, P. Meredith, P.E. Shaw, The nature and role of trap states in a dendrimer-based organic field-effect transistor explosive sensor, Appl. Phys. Lett. 102 (24) (2013), http://dx.doi.org/10.1063/1.4810914, 243301.

[13] R.S. Dudhe, S.P. Tiwari, H.N. Raval, M.A. Khaderbad, R. Singh, J. Sinha, M. Yedukondalu, M. Ravikanth, A. Kumar, V.R. Rao, Explosive vapor sensor using poly (3-hexylthiophene) and Cull tetraphenylporphyrin composite based organic field effect transistors, Appl. Phys. Lett. 93 (26) (2008), http://dx.doi. org/10.1063/1.3049130, 263306.

[14] J. Huang, J. Miragliotta, A. Becknell, H.E. Katz, Hydroxy-terminated organic semiconductor-based field-effect transistors for phosphonate vapor detection, J. Am. Chem. Soc. 129 (30) (2007) 9366-9376, http://dx.doi.org/10. 1021/ja068964z.

[15] L. Torsi, G.M. Farinola, F. Marinelli, M.C. Tanese, O.H. Omar, L. Valli, F. Babudri, F. Palmisano, P.G. Zambonin, F. Naso, A sensitivity-enhanced field-effect chiral sensor, Nat. Mater. 7 (5) (2008) 412-417, http://dx.doi.org/10.1038/ nmat2167.

[16] F. Liao, C. Chen, V. Subramanian, Organic TFTs as gas sensors for electronic nose applications, Sens. Actuators B Chem. 107 (2) (2005) 849-855, http://dx. doi.org/10.1016/j.snb.2004.12.026.

[17] F. Liao, S. Yin, M.F. Toney, V. Subramanian, Physical discrimination of amine vapor mixtures using polythiophene gas sensor arrays, Sens. Actuators B Chem. 150 (1) (2010) 254-263, http://dx.doi.org/10.1016/j.snb.2010.07.006.

[18] L. Torsi, L. a Dodabalapur, P. Sabbatini, Zambonin, Multi-parameter gas sensors based on organic thin-film-transistors, Sens. Actuators B Chem. 67 (3) (2000) 312-316, http://dx.doi.org/10.1016/S0925-4005(00)00541-4.

[19] G. Gu, M.G. Kane, Moisture induced electron traps and hysteresis in pentacene-based organic thin-film transistors, Appl. Phys. Lett. 92 (5) (2008), http://dx.doi.org/10.1016/j.snb.2012.06.060, 053305.

[20] S.H. Kim, H. Yang, S.Y. Yang, K. Hong, D. Choi, C. Yang, D.S. Chung, C.E. Park, Effect of water in ambient air on hysteresis in pentacene field-effect transistors containing gate dielectrics coated with polymers with different functional groups, Org. Electron. 9 (5) (2008) 673-677, http://dx.doi.org/10. 1016/j.orgel.2008.05.004.

[21] A. Das, R. Dost, T.H. Richardson, M. Grell, D.C. Wedge, D.B. Kell, J.J. Morrison, M.L. Turner, Low cost, portable, fast multiparameter data acquisition system for organic transistor odour sensors, Sens. Actuators B Chem. 137 (2) (2009) 586-591, http://dx.doi.org/10.1016/j.snb.2009.01.006.

[22] D. Duarte, D. Sharma, B. Cobb, A. Dodabalapur, Charge transport and trapping in organic field effect transistors exposed to polar analytes, Appl. Phys. Lett. 98 (13) (2011), http://dx.doi.org/10.1063/1.3569592, 133302.

[23] Fraunhofer Institute for Photonic Microsystems, http//www.ipms. fraunhofer.de.

[24] http://fluidat.com, Bronkhorst Fluidat on the Net, 2013.

[25] M. Egginger, S. Bauer, R. Schwödiauer, H. Neugebauer, N.S. Sariciftci, Current versus gate voltage hysteresis in organic field effect transistors, Monatshefte für Chemie - Chem. Mon. 140 (7) (2009) 735-750, http://dx.doi.org/10.1007/ s00706-009-0149-z.

[26] T. Mori, Y. Kikuzawa, K. Noda, Improving baseline stability of gas sensors based on organic field-effect transistors by monitoring carrier mobility, 2011 IEEE SENSORS Proc. (2011) 1002-1005, http://dx.doi.org/10.1109/ICSENS. 2011.6126923.

[27] B. Li, R. Zhang, G. Sauve, J. Cooper, M.C. Iovu, S. Santhanam, L. Schultz, J.L. Snyder, L.E. Weiss, T. Kowalewski, G.K. Fedder, Nanostructure dependence of conductive polymer chemical sensors, IEEE Sensors (2006) 843-846, http:// dx.doi.org/10.1109/ICSENS.2007.355599. 


\section{Biographies}

Peter Lienerth received the Diploma in Physics at the University of Stuttgart in Gemany. After his Master thesis at the Max-Planck-Institute for Metals Research in Stuttgart he received his doctoral degree at the ICUBE laboratory in Strasbourg Fance, where he worked on the characterization of gas-sensing properties of generic and functionalized organic semiconductors.

Sadiara Fall received his master's degree in Micro and Nano Electronics, Physical Components and Systems in 2009 at the University of Strasbourg. He was promoted to Doctor of the University of Strasbourg in April 2013. He did his PhD in the group Organic Photovoltaic Components and Electronics in the Laboratory of Sciences Engineering, Computing and Imaging (ICube) of Strasbourg. His PhD topic was the study of charge transport in organic semiconductors and its impact on the photovoltaic performance. He performed a post-doc in CINaM (Marseille) where he conducted various studies focusing on charge transport, the lifetime of carriers in active layers of solar cells and recombination at interfaces.
Patrick Lévêque received his MSc degree in Material Science in 1994 and his PhD degree in Material Science in 1998 from Poitiers University under the supervision of A. Declémy. He has been docent at the Royal Institute of Technology in Stockholm from 1999 to 2004 and has been assistant professor since then at Strasbourg University. His current research interests include organic electronics with a special focus on charge carrier dynamics in organic semiconductors.

Ugur Soysal received his Master's degree in Nanoscale Engineering at the University Claude Bernard in Lyon in 2015 and worked on chemical sensor during his Master internship at the ICUBE laboratory.

Thomas Heiser obtained his PhD in Semiconductor Physics and Devices from the University of Strasbourg in 1987, where he is working as a faculty member since 1988. Between 1995 and 1997, he joined the University of California in Berkeley as a visiting scientist. In 2000, he has been appointed professor at the University of Strasbourg and is a member of the ICUBE laboratory. His current research interests include charge transport in organic semiconductors and devices, organic solar cells, and chemical sensors based on organic field effect transistors. 